\title{
El Habitus del Profesor de Historia y Ciencias Sociales en Contextos de Pobreza: una perspectiva biográfico/narrativa sobre el Trabajo Docente*
}

\author{
The Habitus of the History and Social Sciences Teacher in Poverty Contexts: a biographi- \\ $\mathrm{cal} /$ narrative perspective on Teaching Work
$O$ habitus do professor de História e Ciências Sociais em contextos de pobreza: Uma perspectiva biográfica/narrativa sobre o Trabalho Docente

\section{Felipe Zurita Garrido}

Academia de Humanismo Cristiano, Escuela de Educación. Condell 506, Providencia, Santiago. Telf.: (56-

02) 7878041. Correo electrónico: fzurita@academia.cl

\begin{abstract}
RESUMEN
Se presentan resultados de una investigación centrada en analizar la construcción simbólica, entendida como Habitus, que realizan los Profesores de Historia y Ciencias Sociales sobre el Trabajo Docente en Contextos de Pobreza. Para dichos efectos, se trabajó con seis docentes que trabajaban en Establecimientos Educacionales ubicados en Contextos de Pobreza de Santiago, realizando entrevistas narrativas que vinculaban la perspectiva que estos actores tienen sobre el Trabajo Docente con su experiencia vital. Los resultados se organizan en las categorías de Trayectoria Profesional, Puesto de Trabajo, Trabajo Docente, Trabajo Docente en Contextos de Pobreza y Profesor/a de Historia y Ciencias Sociales. En las conclusiones se discuten las implicancias que producen las características del Trabajo Docente en los profesores y se proyecta la necesidad de relacionar los resultados de las investigaciones sobre el Trabajo Docente con las discusiones sobre la formación inicial del profesorado y la calidad de la educación chilena.
\end{abstract}

Palabras clave: profesores de historia y ciencias sociales, trabajo docente, habitus, contextos de pobreza.

\begin{abstract}
Here are presented the results of an investigation centered in analyzing the symbolic construction, understood as Habitus, that the History and Social Sciences Teachers do about the Teaching Work in Poverty Contexts. In order to do so, it has been working with six teachers that used to work in educational settings located in Poverty Contexts in Santiago; doing narrative interviews that linked the perspective that these actors have about the Teaching Work and their vital experience. The results are organized in the following categories; Professional Course, Workstation (Job), Teaching Work, Teaching Work in Poverty Contexts and History and Social Sciences Teacher. In the findings are argued the implications produced by Teaching Work's characteristics in teachers and the need of relating the results of the investigations about the Teaching Work with the discussions about the initial training of teachers and education's quality are mapped out.
\end{abstract}

Key words: history and social sciences teachers, teaching work, habitus, poverty contexts.

\section{RESUMO}

Apresentam-se os resultados de uma pesquisa focada em analisar a construção simbólica, entendida como "Habitus", que realizam os Professores de História e Ciências Sociais sobre o Trabalho Docente em Contextos de Pobreza. Para tal, trabalhou-se com seis docentes que atuavam em Estabelecimentos Educacionais localizados em Contextos de Pobreza de Santiago, por meio de entrevistas narrativas que vinculam a perspectiva que estes sujeitos têm sobre o Trabalho Docente com sua experiência vital. Os resultados organizam-se nas categorias de Trajetória Profissional, Local de Trabalho, Trabalho Docente, Trabalho docente em Contexto de Pobreza e Professor(a) de História e Ciências Sociais. Discutem-se as implicações que produzem as características do Trabalho Docente nos professores e projeta-se a neces-

* $\quad$ Este artículo presenta los resultados de una investigación asociada al Proyecto de Investigación CONICYT No 1090692 "Ser docente en Chile: tensiones históricas y perspectivas a través del enfoque biográfico y etnográfico (1923-2011)". 
sidade de relacionar os resultados das pesquisas feitas sobre o Trabalho Docente com as discussões acerca da formação inicial do professorado e a qualidade da educação chilena.

Palavras chave: professores de história e ciências sociais. trabalho docente. habitus. contextos de pobreza.

\section{INTRODUCCIÓN}

En los últimos veinte años se viene debatiendo cada vez con más fuerza en Chile sobre el sistema educacional en su conjunto, tanto así que en dicho periodo el ordenamiento del mismo ha cambiado de manera relevante gracias a diversas transformaciones impulsadas por el Estado, dentro de las cuales resalta con mayor notoriedad la Reforma Curricular de mediados de la década de los noventa y el nuevo marco regulatorio surgido al alero de la Ley General de Educación (2009). Dicho énfasis en torno a reflexionar y emprender transformaciones sobre el ordenamiento del sistema educacional responde a los intentos sistemáticos del Estado chileno de atender las demandas que diversos actores, desde dentro y desde afuera, ejercen sobre el mismo a propósito del tipo de sociedad a construir a través de la instancia educativa. Las demandas enarboladas desde adentro han correspondido a aquellas que diversos actores, como las Universidades, el Colegio de Profesores, la Confederación de la Producción y del Comercio, las Fuerzas Armadas, la Iglesia Católica, la Masonería, Estudiantes Secundarios y Universitarios, Padres y Apoderados, Municipalidades, Dueños de Colegios Particulares, Centros de Investigación, entre otros, han puesto sobre la mesa, en diversos momentos y con diferentes intereses, en la discusión sobre el tipo de educación que Chile debería tener. Sobre las demandas emitidas desde afuera es posible nombrar a aquellos grandes lineamientos educacionales que instituciones internacionales han situado como las vías más apropiadas para encaminar los esfuerzos de formación de las nuevas generaciones, dentro de éstos sobresale la propuesta de CEPAL-UNESCO (1992) Educación y Conocimiento: eje de la transformación productiva con equidad que vincula de manera insoslayable al sistema educativo con el aparato productivo bajo la promesa de la superación de los males económicos y políticos estructurales de América Latina si es que ésta logra encaminarse en el proyecto de modernización neoliberal en su versión globalizante.

Estas demandas desde adentro y desde afuera podrían establecerse también en una escala jerárquica organizando a las mismas bajo la lógica desde arriba y desde abajo en términos de su real influencia y atención prestada por parte de las instituciones estatales que definen los lineamientos a seguir por el sistema educativo. En esta dirección, sobre quién finalmente ha hecho pesar su sentir en el establecimiento de los principales lineamientos de la educación chilena, no resulta muy aventurado señalar que quizás el profesorado ha resultado ser, por un lado, uno de los actores educativos sobre quien más se ha discutido desde una amplia perspectiva, tocando temáticas variadas como por ejemplo el tipo de formación inicial y continua deseable, las estrategias para calificar su desempeño (evaluación docente), la definición de sus potenciales integrantes, la evaluación a las instituciones formadoras de docentes, el tipo de remuneración a recibir, estrategias nacionales e internacionales de asignación de incentivos, entre otras. Mientras que por otro lado, viene constituyéndose cada vez con más claridad en un actor desoído o deslegitimado como referente a considerar en el establecimiento de las mismas medidas administrativas, pedagógicas y políticas que tienen que ver directamente con su trabajo. Esta situación de desentendimiento con respecto a la mirada y postura que el profesorado 
construye sobre su trabajo y su función sociopolítica potencialmente puede generar un distanciamiento abismante entre lo que las políticas públicas decretan y difunden a través de sus distintos medios de comunicación y lo que ocurre realmente en las prácticas pedagógicas cotidianas desarrolladas en las escuelas y liceos del país. Por otra parte, con el paso del tiempo dicho distanciamiento se ha ido naturalizando de tal forma que en el discurso público del Estado de Chile y de otros actores vinculados al sistema educacional resulta difícil identificar una estrategia de simulación u ocultamiento de la creencia de que el mejor camino es hacer reformas y tomar medidas de contingencia educacionales sin considerar la opinión del profesorado. La situación comentada hasta aquí se presenta como un gran desafío para quienes se dedican a la enseñanza escolar, a investigar en el ámbito educacional y también a aquellos que trabajan en la formación de los futuros docentes, puesto que surge el cuestionamiento sobre si es posible avanzar en torno a una reflexión seria, legitimada, propositiva y contextualizada sobre el tipo de educación que como sociedad se quiere y necesita sin enfocarse en la figura central del profesor que finalmente porta en sí mismo el mandato social de cristalizar un tipo de sociedad mejor a partir de su trabajo. Basándose en lo planteado anteriormente, esta investigación da cuenta de la mirada de dicho actor, puesto que el tema a abordar es el Habitus construido por parte de los Profesores de Historia y Ciencias Sociales sobre el Trabajo Docente en establecimientos educacionales ubicados en Contextos de Pobreza. De esta manera, se intentará realizar un acercamiento cualitativo a la manera en que dichos actores conciben el Trabajo Docente a partir de la reflexión sobre su trabajo cotidiano, situado además en un contexto educacional que presenta ciertas características que complejizan al mismo. Desde esta perspectiva, el punto central a abordar en esta investigación es la construcción simbólica que hacen los Profesores de Historia y Ciencias Sociales sobre el Trabajo Docente en establecimientos educacionales ubicados en Contextos de Pobreza.

Se ha optado por realizar esta investigación sobre Profesores de Historia y Ciencias Sociales que ejerzan en establecimientos educacionales ubicados en Contextos de Pobreza de la ciudad de Santiago debido a la directa vinculación del campo de conocimiento abordado por este segmento de Profesores, la Historia y las Ciencias Sociales, con los problemas políticos y sociales que viven los actores educativos pertenecientes a dichas instituciones y en dichos territorios, por ende, es posible hablar de una intima convivencia entre actores, contenido y contexto, relación que se construye en un entramado de significados posibilitadores y limitantes de instancias y esfuerzos transformativos y reproductores de la misma, ya sea a nivel simbólico o real.

\section{HABITUS}

El concepto de Habitus es el utilizado en esta investigación para dar cuenta de los procesos de construcción simbólica que realizan los Profesores de Historia y Ciencias Sociales sobre su Trabajo. Para abordar la definición de dicho concepto se trabajará en base a las aportaciones teóricas que ha realizado el sociólogo francés Pierre Bourdieu en torno a la Teoría de la Acción. Esta selección se justifica en el argumento de que la propuesta de análisis de Pierre Bourdieu apunta a visualizar el proceso de construcción de la forma de comprender la realidad desde una perspectiva que conjuga lo objetivo y lo subjetivo, en otras palabras, muestra que el pensamiento y la acción de los sujetos no se define ni por la articulación reflexiva/mental de los sujetos ni tampoco por las 
estructuras sociales o económicas por sí solas, se trata, por el contrario, de un completo proceso de articulación de estas dos dimensiones actuando dialécticamente. El concepto de Habitus propuesto por Pierre Bourdieu (1997) puede abordarse a partir de la siguiente argumentación:

Más generalmente, el espacio de las posiciones sociales se retraduce en un espacio de tomas de decisión a través del espacio de las disposiciones (o de los habitus); o, dicho de otro modo, al sistema de desviaciones diferenciales que define las diferentes posiciones en las dimensiones mayores del espacio social corresponde un sistema de desviaciones diferenciales en las propiedades de los agentes (o de las clases construidas de agentes), es decir en sus prácticas y en los bienes que poseen (1997: 19).

Desde la perspectiva del autor, el Habitus correspondería al sistema de desviación $o$ aficiones vinculadas a diferentes clases de personas. Este sistema de orientación de aficiones y prácticas es producido por los condicionamientos sociales que afectan a los diferentes grupos de agentes. Entendido así, el Habitus se constituiría en un organizador de las formas de operar de los agentes en base a su posición específica en el campo, lo que se traduciría en una presencia correlacional de las prácticas o aficiones de los agentes, ya sea individuales o grupales, dependiendo de su pertenencia a una clase o grupo en específico. A partir de las propuestas de Pierre Bourdieu (1997) es posible entender al Habitus como el proceso de comprensión de la realidad social y de sí mismo realizada por los sujetos desde una perspectiva centrada en la tensión entre, por un lado, las formas tradicionales y legitimadas de percepción correspondientes a su clase y condición social/ cultural y, por otro lado, la vinculación colaborativa y conflictiva con otros sujetos en un contexto marcado por posicionamientos de orden económico y cultural que dispone una estrategia relacional definida por correlaciones de fuerzas no estáticas. En este sentido, el Habitus se articula como la instancia de otorgamiento de sentido a la realidad por parte de los sujetos en medio de la tensión entre las vivencias de los sujetos y su localización de los mismos en la sociedad, desde esta perspectiva, dicho otorgamiento de sentido no pasa solamente por la estructura cognitiva de los sujetos y las experiencias simbólicas individuales de los sujetos, como así tampoco solamente por la determinación de los condicionamientos económicos y sociales, por el contrario, en la configuración del Habitus interactúan la dinámica social, económica y cultural entendidas en una perspectiva de larga data, o sea histórica, que han pasado en parte a corporizarse en las diferentes clases sociales junto a un componente creativo y revolucionario de los sujetos, articulado de acuerdo a las lecturas y posicionamiento concretos que dinámicamente van asumiendo en un contexto social concreto en constante pugna entre la reproducción y la transformación.

\section{EL CONCEPTO DE TRABAJO EN MARX}

Si bien Carlos Marx no realiza una definición específica sobre el concepto de Trabajo en sus escritos, José Noguera (1998) señala que a partir de otras definiciones y teorizaciones, construidas por el mismo autor, sí es posible reconstruir una perspectiva específica sobre este concepto. Esta tarea de reconstrucción teórica de la noción de Trabajo de Carlos Marx incluye la idea central de que este se define como un concepto amplio debido a que "(...) va más allá de la pura instrumentalidad y posibilita una vía de autorrealización del ser humano” (José Noguera, 1998: 49). Para justificar esta afirmación 
se revisarán tres dimensiones vinculadas a la definición de Trabajo de Carlos Marx que permitirán comprender esta perspectiva de concepto amplio: instrumentalidad, socialidad y expresividad. Sobre la dimensión de instrumentalidad del Trabajo en Carlos Marx, José Noguera (1998) señala que efectivamente para este autor el Trabajo tiene una perspectiva orientada hacia fines concretos establecidos:

El trabajo es una actividad orientada a un fin, y por ende, con arreglo al aspecto material, está presupuesto que en el proceso de producción el instrumento de trabajo sea utilizado realmente como un medio para un fin. (Carlos Marx, 1857-1858: 251 en José Noguera, 1998: 49).

Asumiendo la existencia de estos fines del Trabajo, José Noguera (1998) señala que los mismos no quedan circunscritos a un nivel de conciencia, sino que se materializan en el mundo al construir o transformar objetos. Reconociendo este carácter instrumental del Trabajo en Carlos Marx, resulta necesario señalar que su conceptualización del mismo no queda limitada a dicho carácter, puesto que el hombre no sólo trabaja con miras a satisfacer necesidades, sino que se trataría de una actividad que dinamiza e incluye otras dimensiones:

El trabajo es, en primer lugar, un proceso entre el hombre y la naturaleza, un proceso en que el hombre media, regula y controla su metabolismo con la naturaleza (...) a fin de apoderarse de los materiales de la naturaleza de una forma útil para su propia vida. Al operar por medio de ese movimiento sobre la naturaleza exterior a él y transformarla, transforma a la vez su propia naturaleza. Desarrolla las potencias que dormitaban en ella y sujeta a su señorío el juego de fuerzas de la misma (Carlos Marx, 1872: 215-216 en José Noguera, 1998: 51).

Con respecto a la dimensión de socialidad en la conceptualización de Trabajo en Carlos Marx se puede señalar que efectivamente existe un claro carácter social debido a que en el mismo el hombre construiría su identidad y la auto-conciencia. Esta afirmación se sustenta en la visión de hombre que defiende Carlos Marx al entender a este como un Animal Social imposible de ser analizado fuerza de la relación e interacción con otros:

Individuos que producen en sociedad, o sea la producción de los individuos socialmente determinada: este es naturalmente el punto de partida. El cazador o el pescador sólos y aislados, con los que comienzan Smith o Ricardo, pertenecen a las imaginaciones desprovistas de fantasía que produjeron las robinsonadas dieciochescas (Carlos Marx, 1857-1858 en José Noguera, 1998: 55).

En la cita anterior es posible identificar una perspectiva de socialidad radical en Carlos Marx sobre el Trabajo, descartando con fuerza la posibilidad de acercarse a una comprensión individualizada del hombre en el análisis del funcionamiento del sistema productivo. Por el contrario, para Carlos Marx el mismo Trabajo genera condiciones de encuentro y cooperación entre los hombres, puesto que tanto las necesidades como también las actividades asociadas a su satisfacción son sociales, compartidas, ya que la sociedad es finalmente un horizonte insuperable para la acción humana. Incluso, cuando se hace referencia de una actividad individual, en solitario, dicha labor se inserta de igual manera en un contexto social.

Por otra parte, sobre la dimensión de expresividad del concepto de Trabajo en la obra de Carlos Marx se puede señalar que existirían algunos elementos interesantes dentro de su perspectiva sobre la misma vinculados a la potencialidad estética y expresiva del hombre.

Como ya se ha señalado con anterioridad, para Carlos Marx a través del Trabajo el hombre transforma la naturaleza y se transforma a sí mismo, también satisface sus 
necesidades y humaniza a la naturaleza. Esta perspectiva sobre el Trabajo reconoce la dimensión utilitaria del Trabajo (satisfacer necesidades), inserta en un contexto social y a la vez incluye el potencial creador y auto-creador humano del mismo, articulado por supuesto por la capacidad expresiva y creativa del hombre. Sobre este punto, Carlos Marx articula una crítica al Trabajo en el contexto del Capitalismo al señalar que en este se produce la enajenación de una parte del ser humano al limitarse el Trabajo solo a la dimensión instrumental del mismo. En esta línea, se puede señalar que “(...) aparece la realización del trabajo como desrealización del trabajador”. (Carlos Marx, 1844: 106 en José Noguera, 1998: 61). La argumentación propuesta hasta aquí en torno a la potencialidad expresiva y creativa del hombre a través del Trabajo permite visualizar una interesante vinculación entre Trabajo y Placer en la perspectiva de Carlos Marx puesto que suma a las dimensiones instrumental y social revisadas hasta aquí, una dimensión expresiva que establece un lazo entre Trabajo y Placer, entre Trabajo y Autorrealización. Esta nueva dimensión del Trabajo en Carlos Marx permite ampliar la conceptualización del mismo hacia una esfera que facilita ligarlo a instancias que potencian y valoran la humanización de la actividad del hombre, al hablar de humanización también se debe incluir la viabilidad de la expresión de la autenticidad del hombre, en este punto el Trabajo es quizás la instancia privilegiada para la misma.

\section{TRABAJO DOCENTE}

En este escrito se sostiene una conceptualización del Trabajo Docente que recoge algunas de las principales aportaciones de la definición de Carlos Marx sobre el Trabajo. En esta línea, es posible señalar que en esta investigación se ha optado por construir una mirada sobre el Trabajo Docente coherente con el interés de estudiar la actividad docente desde la perspectiva de quien lleva a cabo y vivencia los diferentes matices de la misma. De esta forma, se sostiene que la propuesta que se presentará a continuación cumple con dichos criterios, puesto que se define al Trabajo Docente como la actividad realizada por profesores y profesoras en el contexto del sistema educativo con miras a la promoción cultural, social y afectiva de las nuevas generaciones, por lo mismo, se trata de un trabajo de creación e intervención constante en la realidad de otros y en la propia. Desde la perspectiva anterior el Trabajo Docente es una actividad, dentro de otras más, que pretende humanizar las relaciones entre las personas con miras a generar las condiciones adecuadas para la promoción de todos los que participan en el proceso formativo escolar, incluidos los mismos docentes. Esta actividad realizada entre y para personas está mediada por la cultura o por parte de la cultura que se ha definido socialmente como aquella digna de ser transmitida de generación en generación en la instancia educativa escolar, por lo mismo, es posible señalar que el Trabajo Docente es una actividad inscrita en el área del Trabajo Cultural.

Sumado a lo dicho hasta aquí, también se sostiene que el Trabajo Docente incluye las dimensiones del concepto de Trabajo planteadas por Carlos Marx: en primer lugar, es una actividad de carácter instrumental puesto que quienes la desempeñan buscan a través de la misma asegurar condiciones materiales adecuadas para la sobrevivencia biológica, por ende, la desarrollan en base a la condición de trabajador asalariado y en ese contexto persiguen una mejora constante de las condiciones de intercambio entre ellos y un 
empleador. Es instrumental también porque persigue finalidades específicas que orientan su accionar. En segundo lugar, el Trabajo Docente es una actividad que tiene una dimensión social evidente puesto que se desarrolla en un contexto institucional donde quizás se asegura de manera más radical el encuentro obligatorio, sistemático y sostenido en un tiempo prolongado de las personas en la sociedad. El Trabajo Docente se desarrolla en un contexto institucional: la Escuela y el sistema educativo en general, situación que exige una comprensión contextualizada de las condiciones (posibilidades y limitantes) que encuentra normativamente dicha actividad en la realidad. Por otra parte, el Trabajo Docente es parte fundamental del funcionamiento de la sociedad, puesto que es un factor relevante dentro de la articulación de las condiciones culturales, políticas e ideológicas de la reproducción como también del cambio social a través de la instancia educativa. En tercer lugar, el Trabajo Docente posee una dimensión de expresividad potentísima, puesto que demanda de quien lo ejerce la vinculación de todo su potencial intelectual, creativo y afectivo. Dicho potencial también se debe entender en una dirección orientada hacia la autorrealización de los profesores y profesoras en y a través de su actividad laboral, ya que la actividad emprendida por dichos actores intrínsecamente está dirigida hacia el logro del bienestar intelectual y emocional de toda la comunidad educacional. Ciertamente, la dimensión expresiva del Trabajo Docente amerita una especial atención, ya que al incluirla como parte fundamental de la definición de este concepto se realiza a la vez una invitación a indagar en la específica relación entre Trabajo y Placer en la docencia. En esta misma dirección, es preciso señalar la necesidad de la autonomía en el desarrollo del Trabajo Docente puesto que se trata de una profesión, tipología de actividad caracterizada por contar con instancias institucionales de formación socialmente legitimadas que velan por el adecuado funcionamiento y operacionalización de procesos académicos que culminan en la obtención de un Título Profesional. El Título Profesional es finalmente la coronación de cierto estatus intelectual y técnico de quien lo posee y que lo inviste de cierta autonomía, por ende, el Trabajo Docente con miras a la autorrealización se caracteriza por el aseguramiento del accionar autónomo y socialmente valorado.

La actividad realizada por los profesores y profesoras, el Trabajo Docente, se entiende en esta investigación también desde una perspectiva más concreta vinculada a la noción de Puesto de Trabajo, que corresponde a las características más específicas que configuran la vivencia de dicha labor. El Puesto de Trabajo se vivencia también a través de las tres dimensiones del concepto de Trabajo analizadas con anterioridad. En un intento de describir los componentes del Puesto de Trabajo se podría incluir a diversas actividades y condiciones a considerar que no pueden limitarse solamente al trabajo de aula, puesto que esa perspectiva es un tanto reduccionista debido a que las actividades emprendidas por el profesorado en el ámbito laboral implican un abanico de tareas bastante más amplias donde caben: la preparación de la enseñanza; la evaluación constante sincrónica y diacrónica del proceso y resultados de la enseñanza; la organización e implementación de actividades extracurriculares con los estudiantes; la organización e implementación de actividades con las familias y la comunidad a la que pertenecen los estudiantes y la institución escolar; la organización e implementación de actividades institucionales o festividades cívicas o religiosas dentro de la institución escolar; la organización e implementación de actividades de corte sindical y gremial que apuntan a mejorar las condiciones de trabajo y salarios tanto dentro de la institución escolar como así también a nivel local y nacional; el desarrollo de actividades personales e institucionales de perfeccionamiento 
disciplinar y pedagógico; el desarrollo de actividades administrativas a distintos niveles tanto al interior de la institución escolar como a niveles más amplios como la comuna o ciudad de pertenencia; la vinculación a grupos de profesores organizados de corte gremial/ artístico/social/político/disciplinar en distintos niveles de escala territorial; el desarrollo de actividades de orientación y guía a los estudiantes y sus familias tanto en momentos emergentes como de forma sistemática; el emprendimiento de acciones que apunten a influir en el desarrollo e implementación de Políticas Públicas vinculadas a su quehacer y entre muchas otras.

Dentro de las condiciones de desarrollo del Puesto de Trabajo se pueden señalar el nivel de remuneraciones alcanzado en un momento determinado, la cantidad de horas de trabajo en aula y fuera de ella incluidas en el Contrato de Trabajo, la cantidad de horas de dedicación a actividades pedagógicas realizadas fuera de la jornada de trabajo remunerado, la cantidad de horas invertidas para trasladarse al lugar de trabajo y al hogar, la extensión del Contrato de Trabajo, la disposición de infraestructura y materiales necesarios para el desarrollo de diversas actividades dentro de la institución educativa, las proyecciones laborales existentes en la institución educacional donde se trabaja, las relaciones sindicales/laborales con el empleador, la existencia de procedimientos de reconocimiento simbólico y material al trabajo realizado, los niveles de autonomía o sujeción en el desempeño del trabajo pedagógico que entrega la institución educacional, niveles de satisfacción o malestar asociados al desempeño del trabajo cotidiano, las relaciones humanas a todo nivel (entre profesores, entres profesores y directivos, entre profesores y estudiantes, entre profesores y apoderados, etc.), entre muchas otras.

En el caso específico del Puesto de Trabajo del profesorado chileno, se puede señalar que, en términos de las condiciones laborales que estructuran al mismo, se cuenta con una regulación diferenciada, puesto que los docentes que trabajan en Establecimientos Educacionales de Dependencia Municipal se encuentran amparados por el Estatuto Docente que rige condiciones legales mínimas y específicas sobre el Trabajo Docente en dicho contexto, mientras que los profesores que trabajan en Establecimientos Educacionales Particulares Subvencionados y Particulares Pagados cuentan con el Código del Trabajo como instrumento legal para organizar sus actividades, aunque este corresponde a definiciones genéricas para el colectivo de trabajadores de Chile. El Estatuto Docente, en su diseño, también está considerado para los profesores que trabajen en Establecimientos Educacionales de Dependencia Particular, no obstante, su aplicación completa no es forzosa en dicho contexto, por lo tanto, en la realidad solo se consideran algunos aspectos mínimos del mismo.

En conclusión, existe una diferenciación entre el profesorado chileno en torno al aseguramiento legal de condiciones laborales conocidas, consensuadas y legitimadas. Dicha diferenciación deja al arbitrio de las decisiones de los empleadores y de una legislación laboral en extremo flexible al profesorado que se desempeña en Establecimientos Educacionales Particulares Subvencionados y Particulares Pagados en Chile. Mientras que por otra parte se establecen e implementan normativas que apuntan a establecer con claridad qué es lo que debe y puede realizar el profesorado que se desempeña en Establecimientos Educacionales Públicos o Municipales. Por otra parte, según la OCDE (2009) el Puesto de Trabajo del profesorado chileno se caracteriza por el desempeño con un alto número de estudiantes promedio por sala (30 en Educación Básica y 31 en Educación Media), un bajo nivel de remuneraciones en todos los momentos de la 
Trayectoria Profesional, un alto número de Días Lectivos de Trabajo (191 al año), un alto número de Horas de Trabajo Anual (1.152), un alto número de Horas de Trabajo en Aula de la Jornada Laboral (71\%) en detrimento de las Horas de Trabajo No Lectivas de la Jornada Laboral (29\%). Estos datos ubican a Chile como el país miembro de la OCDE que presenta los índices más altos de intensificación laboral del profesorado (muchos estudiantes que atender, muchas horas de trabajo anual y mayoritariamente realizando actividades de enseñanza de aula) y a la vez con el índice más bajo de remuneración a corto y largo plazo.

Para resumir, el Puesto de Trabajo del profesorado en Chile se caracteriza por: a) una clara diferenciación entre diferentes tipos de profesores, tanto a nivel de las diferentes Dependencias de los Establecimientos Educacionales, b) la dificultad para proyectar las actividades del profesorado más allá de los límites de su sala de clases, debido al establecimiento de limitantes claras para la participación, perfeccionamiento e injerencia clara en la estructuración de su propio Trabajo, c) intensificación laboral y d) el bajo nivel de las remuneraciones percibidas por el profesorado en Chile y por el corto alcance que puede llegar a tener el mismo en el tiempo. Estas características del Puesto de Trabajo impactan potencialmente en los niveles de calidad de vida del profesorado, como así también en las expectativas de los mismos actores en torno a la motivación para perfeccionarse y así poder escalar en la Trayectoria Profesional, como así también en las expectativas para mantenerse en la misma después de un tiempo.

\section{LA CONFIGURACIÓN CONTEMPORÁNEA DEL TRABAJO DOCENTE}

Emilio Tenti Fanfani (2007) aborda una serie de transformaciones sociales que impactan de manera sistemática y profunda al Trabajo Docente. La primera de estas transformaciones es la "(...) masificación de la escolaridad con exclusión social” (2007: 121). El fenómeno de la impresionante (y necesaria) ampliación de la escolaridad ha transformado la faz de las sociedades que han asumido dicha tarea con fuerza y decisión. Las transformaciones más relevantes dicen relación con la extensión de la alfabetización, la democratización del acceso a la educación, la complejización de la estructura ocupacional y profesional, la facilidad de acceder a la información y participar social y políticamente de manera activa, en pocas palabras, el trabajo por forjar una incorporación al mundo social a través de la instancia educativa, desde un punto más equitativo desde los primeros años de vida.

Reconociendo el impacto profundamente valioso y necesario de la ampliación de la escolaridad, también es necesario reconocer que por supuesto dicho proceso ha sido desigual dependiendo de cuán segregada y desigual es la sociedad y sistema educacional analizado. De esta manera, el Trabajo Docente contemporáneo se caracteriza por la fuerte implicación de los docentes con su actividad, tornando borrosa la frontera entre trabajo y vida personal; por otra parte, está la tensión entre el igualitarismo académico y las características desiguales de los estudiantes, tensión que idealmente se enfrenta al asumir la complejidad de la labor docente y a una adecuada preparación pedagógica y didáctica que le permita a los docentes enfrentar de mejor manera las demandas de diverso tipo que implica el Trabajo Docente contemporáneo (Emilio Tenti Fanfani, 2007).

La segunda transformación social que impacta al Trabajo Docente dice relación con "Los cambios en las relaciones de poder entre las generaciones y en la estructura y 
dinámica de los grupos familiares" (Emilio Tenti Fanfani, 2007: 125). Las transformaciones de poder en las relaciones entre las viejas y nuevas generaciones han mermado la configuración clásica de relación entre docentes y estudiantes, los segundos ahora son sujetos poseedores de derechos desconocidos hace algunas décadas atrás y por lo mismo comparten una comprensión de esta nueva situación que les permite demandar una vinculación más respetuosa con ellos por parte de la Escuela; de esta manera, las nociones de autoridad docente y orden que facilitaban la enseñanza se han deteriorado, empujando a docentes y estudiantes a pugnar y cooperar en la creación de nuevos dispositivos que orienten la relación entre ambos dentro y fuera de la sala de clases. Sumado a esto es necesario incluir al surgimiento de las culturas juveniles que se constituyen en un fenómeno que torna compleja la relación con los docentes, principalmente por la diferencia generacional, debido a que estos no comprenden ni legitiman los valores que estos portan y viven, situación que puede leerse también por parte de los estudiantes.

En un segundo momento, hay que señalar que las transformaciones en la composición familiar juegan un rol fundamental en la manera en que el Trabajo Docente se desarrolla en la actualidad; de manera tradicional la Escuela se pensó funcionando en asociación con la familia, existía una división de tareas a cumplir en la formación de los niños y jóvenes, no obstante, dicho "pacto" se ha quebrado, principalmente por las complicaciones que presentan las familias para hacerse cargo de este rol tradicional en un contexto de transformaciones económico-laborales que cercenan la composición clásica de la familia (madre dueña de casa-hombre trabajador) y empujan a los adultos del grupo familiar a trabajar en jornadas y horarios que no facilitan el acompañamiento y dedicación del proceso educativo de sus hijos. De esta manera, la Escuela y los docentes deben asumir la formación de niños y jóvenes responsables de sí mismo, sin adultos como soporte material y afectivo. También existen familias que por el contrario revisan, vigilan y cuestionan de cerca el quehacer docente. De esta manera, según los docentes, existiría un fenómeno nuevo de falta de involucramiento o intervención excesiva por parte de las familias, lo que dificulta su Trabajo cotidiano. Este nuevo marco de acción caracterizado por la imprecisión de las tareas familiares y pedagógicas sitúa al Trabajo Docente en un nivel de complejidad que impacta en la autopercepción y motivación de los docentes, lo que “(...) con frecuencia produce una sensación de malestar e impotencia que inevitablemente afecta la identidad profesional de los trabajadores de la educación". (Emilio Tenti Fanfani, 2007: 127).

Finalmente, la tercera transformación que impacta al Trabajo Docente contemporáneo apunta a "Las innovaciones en el campo de las nuevas tecnologías de la información y la comunicación". (Emilio Tenti Fanfani, 2007: p. 128). Las conocidas transformaciones en este campo han llegado con fuerza a las Escuelas, no obstante, esta llegada se ha caracterizado por la imposición dogmática de la idea de que a más computadores y tecnología en general en la Escuela, más avanzada y mejor es la educación. Sin embargo, dicha "llegada" no ha ido acompañada de una preocupación seria con respecto a cómo incluir y vincular a los docentes en este nuevo ámbito; de hecho, en muchos casos son los estudiantes quienes manejan con más soltura la utilización de estas nuevas tecnologías.

Las transformaciones precedentes invitan a pensar en torno a cómo se desarrolla la pugna por la definición del Trabajo Docente según los diferentes momentos históricos abordados. Para Emilio Tenti Fanfani (2007) es posible diferenciar dos momentos de los contextos organizacionales que condicionan el Trabajo Docente: el primero es el de "Burocracia Educativa" situado en el inicio de la formación de los Sistemas Educacionales 
Nacionales; allí el Trabajo Docente se caracterizó por la doble y confusa filiación a las figuras de apóstol y funcionario, caracterizado por altas demandas de dedicación al trabajo y demostración de características ético-morales adecuadas para asumir el mismo. En este contexto, el Trabajo Docente es vivamente vigilado y normado, no poseen el control sobre su actividad, se van perfilando como una "semi-profesión" y los maestros se mostraban muy comprometidos con la misión educacional a través de la ideología de la vocación, de la cual participaban y legitimaba su decisión profesional. En este contexto la constitución de una identidad gremial es compleja y lenta, principalmente por la heterogeneidad del cuerpo docente. El segundo momento organizacional es definido como "Post-Burocrático" y se ubica en el momento histórico de crisis del Estado Nacional hasta la actualidad. En este contexto, el Trabajo Docente ha visto entrar en crisis los principios que lo estructuraban en la época precedente, principalmente por la puesta en marcha en el seno del funcionamiento de los sistemas educacionales a "(...) los nuevos modelos de organización característicos de las organizaciones más dinámicas del capitalismo moderno" (Emilio Tenti Fanfani, 2007: 132-133). En esta línea, para dar cuenta de esta transformación Emilio Tenti Fanfani (2007) toma las ideas de Gilles Deleuze (1990) sobre las sociedades de control y de Luc Boltansky y Eve Chiapello (1999) sobre el nuevo espíritu del capitalismo. Sobre la primera idea se señala que las organizaciones disciplinarias tradicionales, caracterizadas por la dominación directa a través del control espacial y temporal de la actividad de los sujetos, han sido reemplazadas por otros dispositivos que terminan ejerciendo un efecto estructural en los mismos. También las organizaciones productivas han dejado de ser las grandes unidades pesadas y fuertemente estructuradas, donde los sujetos trabajaban mediante mandatos impuestos centralizadamente, por el contrario, el sistema productivo en la actualidad se caracteriza por la atomización y funcionamiento en red de pequeñas unidades articuladas en torno a proyectos elaborados con cierta autonomía programática y financiera que funcionan en pos de obtener resultados específicos a evaluar al término del proceso. De esta manera, la presencia de márgenes de libertad y ausencia del tradicional control/obligación directa generan un nuevo marco a través del cual los trabajadores se involucran de una nueva manera con su oficio. La segunda idea tomada por Emilio Tenti Fanfani (2007) dice relación con la asunción de un nuevo espíritu del sistema capitalista en su actual fase de desarrollo, que apunta a los trabajadores a implicarse de manera sistemática e intensiva en las unidades productivas. Dicha implicación se produciría por la ruptura con el fordismo debido a la recuperación "(...) de una parte del sentido de su trabajo" (133) gracias a la extensión del Trabajo en equipo y en base a proyectos donde se difumina la tradicional lógica de estipulación prescrita y específica de aquello puntual que cada trabajador debe realizar.

\section{TRABAJO DOCENTE EN CONTEXTOS DE POBREZA}

En esta investigación se ha optado por una perspectiva materialista para comprender la producción de la Pobreza en tanto se asume a esta como un fenómeno directamente vinculado al Modelo Económico Capitalista, que implica en sí mismo la producción sin precedentes de Riqueza y Pobreza indisolublemente, mientras que, por otra parte, la situación de ser pobre se entiende aquí desde una perspectiva que exige un análisis y comprensión global de la misma, puesto que dicha condición no responde a una culpa individual como 
se ha asumido desde las propuestas de Adam Smith en adelante en Occidente, sino que por el contrario esta se entiende en una relación dialéctica entre lo económico, lo social y lo político en términos más amplios y complejos (Redondo, 2004). En esta misma línea, Milton Santos (2004) habla de "división 'administrativa' del trabajo" y apunta a establecer que la distribución de la producción económica está organizada a nivel planetario en base a procesos de Verticalidades de carácter coactivo a través de mecanismos diplomáticos/ políticos, ideológicos e incluso militares, por lo mismo, dentro de la misma dinámica de avance sin límites del capital financiero y el despliegue de la ciencia y la técnica por el planeta en su conjunto, va de la mano una organización y distribución de procesos de exclusión conducentes a formas de vida caracterizadas por la Pobreza. Esta acción doble, de producción de Riqueza y Pobreza, se realiza de manera orquestada debido a la participación complaciente y deliberada de los Gobiernos y elites en los diferentes países.

De esta manera, la noción de Pobreza se asocia con la noción de Exclusión, debido a que en el contexto contemporáneo de producción a nivel planetario lo que prima son niveles de acumulación de bienes y servicios gigantescos nunca antes vistos, que por su tamaño alcanzarían sin muchas dificultades para dar cuenta de las necesidades de la población de los cinco continentes, sin embargo, como en el Sistema Económico Capitalista la Mercancía se define por su valor de cambio y no por su valor de uso, parte importante de la población mundial no accede finalmente a dicha producción por no contar con el capital para adquirirlo en el mercado desregulado de cualquier preocupación ética o humanitaria. La Pobreza como Exclusión se entiende entonces como la imposibilidad de acceder a los bienes para satisfacer necesidades materiales y culturales básicas que el mercado posee en abundancia pero que pone límites al mismo deliberadamente. En esta lógica, la Globalización Perversa imperante en la actualidad construye vivencias concretas de Pobreza a través de la acción de Verticalidades en el Espacio Geográfico, donde se establecen formas de vida marcada por la Exclusión a los bienes y servicios necesarios para dar cuenta de las necesidades materiales y culturales básicas de la población, en espacios territoriales amplios. De esta manera, cuando aquí se plantea la noción de Contextos de Pobreza se deben entender como territorios impactados por los efectos de la desigual organización planetaria del trabajo y la decisión racional de los Estados de abandonar su rol regulador de la relación entre el Capital y el Trabajo. Estos Contextos de Pobreza son aquellos territorios caracterizados por la precarización de las condiciones de trabajo de su población, por la presencia paupérrima del Estado como asegurador de las necesidades básicas de la población (salud, vivienda, educación), por la presencia limitada y segmentada de condiciones materiales y culturales para el desarrollo igualitario de las personas, la presencia desbordante y excluyente del mercado como instancia de solución de las necesidades de la población.

En esa dirección, la definición de Contextos de Pobreza no se limita a los parámetros administrativos de cuantificación de los niveles de ingreso monetario de las familias como tampoco a la utilización de grandes territorios entendidos desde una perspectiva homogeneizante como parámetros para definir la Pobreza, por el contrario, aquí se presenta una propuesta que articula lo global y lo local desde una lógica dialéctica puesto que piensa la construcción de los Contextos de Pobreza en base a las lógicas de las relaciones de producción a nivel planetario y en base a realidades económicas y sociales de escalas más pequeñas. En estos Contextos de Pobreza se encuentran Establecimientos Educacionales que se constituyen en micro-territorios que experimentan los vaivenes de los procesos de Fijos y Flujos y de Verticalidades y Horizontalidades a nivel global y local, situación 
que tiene una correlación con la forma de actuar y significar de los sujetos que allí se relacionan puesto que dichas instituciones no se encuentran al margen de estos procesos, por el contrario, son parte central de la articulación y legitimación de estos procesos en la medida que allí se definen formas y contenidos, tradicionales y novedosos, de pensar y actuar en el mundo. Independiente de su ubicación a nivel de ciudad o comuna y del tipo específico de su propiedad, en los Establecimientos Educacionales es posible identificar vestigios materiales y simbólicos propios de la producción y vivencia de un Contexto de Pobreza puesto que las Escuelas y Liceos son parte de las comunidades en las que se ubican, no son parte del paisaje solamente, estas se vinculan además con otros Establecimientos Educacionales y con otros Espacios Geográficos a través del Estado, a través del Mercado, a través de la Sociedad Civil, a través de las proyecciones y expectativas personales e institucionales, como así también de las frustraciones y limitaciones de todo tipo propias de una Globalización Perversa.

\section{MARCO METODOLÓGICO}

La investigación se inscribe dentro del Paradigma Comprensivo/Interpretativo y asume los lineamientos del Enfoque de Investigación Cualitativa. Lo anterior se sustenta en que se pretende comprender el Habitus construido por los Profesores de Historia y Ciencias Sociales en torno al Trabajo Docente en establecimientos educacionales ubicados en Contextos de Pobreza, dicha comprensión se buscará a través de la reconstrucción de la mirada que los propios actores tienen sobre el fenómeno de estudio sin ánimo alguno de establecer lineamientos generales susceptibles de ser generalizados para todos los sujetos antes mencionados. Esta investigación se ubica además en el Enfoque de Investigación de Historias de Vida o Biográfico Narrativo. Ivor Goodson (2004) presenta una perspectiva muy especial sobre las Historias de Vida del Profesorado al perfilar a este tipo de investigación en una senda que busca generar un diálogo entre el análisis de nivel global con el análisis de carácter local, facilitando así una comprensión del campo de acción y reflexión intimo/ particular/subjetivo del profesorado en un contexto mayor que le presenta desafíos, obstáculos y posibilidades que impactan en la forma en que estos actores configuran dicho campo.

En el caso particular de esta investigación, situada bajo el Tipo de Estudio de Historias de Vida, lo que se realizará finalmente es un acercamiento al objeto de estudio definido en la presentación, el Habitus construido por los Profesores de Historia sobre el Trabajo Docente en Establecimientos Educacionales ubicados en Contextos de Pobreza, a través de una estrategia de recopilación y análisis de información coherente con el Enfoque de Investigación de Historias de Vida o Biográfico Narrativo, pero que no será la elaboración de una Historia de Vida o Relatos de Vida. En esta línea, lo que se realizará será el desarrollo de Entrevistas Narrativas a un grupo definido de Profesores de Historia y Ciencias Sociales con miras a develar a través de la misma lo que se ha definido como objeto de estudio a partir del análisis de las entrevistas realizadas.

\subsection{TIPO DE MUESTRA}

En las investigaciones ligadas al Tipo de Estudio de Historias de Vida el establecimiento de los informantes resulta ser un elemento crucial en la definición del sentido 
y amplitud del trabajo a realizar, por lo mismo, resulta fundamental establecer ciertos parámetros organizadores con respecto a quién o quiénes se incluirán en el mismo y de qué manera. De esta manera, el establecimiento de la naturaleza de la muestra a trabajar se desarrollará según la orientación del Muestreo Teórico que deberá corresponder con el siguiente perfil:

a) Ser Profesores/as de Historia y Ciencias Sociales.

b) Estar trabajando actualmente en Establecimientos Educacionales ubicados en Contextos de Pobreza de la ciudad de Santiago.

c) Ser representativos de las diferentes Fases de Desarrollo Profesional: Fase de Inicio o Entrada, fase de Estabilización o Puesta en Cuestión y Fase de Resolución. d) Distribuirse equitativamente entre hombres y mujeres.

Estos cuatro criterios construyen finalmente un abanico de 3 Profesores y 3 Profesoras de Historia y Ciencias Sociales que trabajen en Establecimientos Educacionales ubicados en Contextos de Pobreza a incluir en la investigación.

\subsection{TÉCNICAS DE RECOLECCIÓN DE INFORMACIÓN}

Se ha optado por utilizar uno de aquellos modelos de entrevista que se adaptan mejor a los intereses de la investigación de Historias de Vida como lo es la Entrevista Narrativa. Esta modalidad de entrevista apunta a lograr un tipo de relato de una experiencia de vida del informante con respecto a un área de interés en el que se pueda dar cuenta de manera amplia sobre cómo se desarrolla la historia desde el comienzo hasta el final de manera coherente y relevando los acontecimientos más relevantes según la perspectiva del mismo. La estructura de este tipo de entrevista es la siguiente: se parte por plantear una pregunta generadora de narración que enmarca la orientación y finalidad de la entrevista a la vez que invita al informante a comunicar de manera libre su perspectiva con respecto al área de interés expresada. En un segundo nivel se van proyectando preguntas que apuntan a profundizar algunos momentos de la narración poco claros o detallados. Se finaliza invitando al informante a dar cuenta de vinculaciones teóricas sobre su narración y a realizar un balance sobre lo expresado que genere un tipo de significado totalizador con respeto a lo mismo (Uwe Flick, 2004). De manera más específica, se le realizará 1 Entrevista Narrativa a cada uno de los 6 Profesores y Profesoras de Historia y Ciencias Sociales incluidos en esta investigación. De esta manera, se utilizará a la Entrevista Narrativa con la finalidad de abrir un espacio de recolección de narrativas amplias y diversas que reconozcan el valor de la intencionalidad de esta técnica de recolección de información, así como también el aporte para generar los imprescindibles resguardos de credibilidad de toda investigación cualitativa y esta en particular.

\subsection{TÉCNICAS DE ANÁLISIS DE INFORMACIÓN}

Encaminándose en el horizonte de generar un tipo de análisis de sea capaz de dar cuenta de la doble preocupación de no dejar intactos los relatos y no intervenirlos en demasía, la presente investigación se inclina por la propuesta de Análisis Temático o de Codificación Temática. Esta estrategia de análisis permite el desarrollo de un trabajo de acercamiento a las Historias de Vida de los sujetos investigados donde sea posible dar cuenta de un esfuerzo de hacer a las mismas inteligibles, según los objetivos de la 
investigación, y comparables, en pos de facilitar un diálogo articulado entre estas y la información que proporcionan. Por otra parte, la Codificación Temática genera instancias de análisis que van señalando un camino adecuado entre los extremos del textualismo radical y el cercenamiento del relato de los entrevistados por parte del investigador. De manera distinta, la Codificación Temática se presenta como un procedimiento de análisis escalonado o de pasos múltiples que permite acercarse a la experiencia individual y colectiva de los sujetos investigados y que facilita llevar adelante una esfuerzo analítico donde es posible conocer de la voz de los profesores incluidos en la investigación, como así también conocer y orientarse a través de los intereses temáticos que la misma investigación posiciona. Los pasos de la Codificación Temática son los siguientes:

a) Realización de una descripción de cada uno de los casos incluidos en la investigación.

b) Construcción de categorías de análisis para cada uno de los casos de manera individual.

c) Desarrollo de un análisis temático cruzado.

d) Construcción de una estructura temática a partir del análisis temático cruzado.

e) Realización de análisis sobre pasajes individuales.

\section{RESULTADOS}

\subsection{SOBRE LA TRAYECTORIA PROFESIONAL}

Los 6 docentes se encuentran en alguna de las tres Fases del Desarrollo Profesional. Dentro de estos, los docentes muestran niveles más consolidados de seguridad sobre sí mismo y sobre su Trabajo al avanzar en edad. Por otra parte, los docentes que más cuestionan su decisión de dedicarse a la Pedagogía son aquellos que han tenido una mayor inestabilidad laboral, lo que coincide con el grupo más joven de los mismos. Justamente, el grupo más joven de los docentes se ha insertado en Programas de Postgrado, con miras a mejorar su estabilidad laboral, remuneraciones y prestigio. Los Programas de Postgrado elegidos se vinculan con actividades de Dirección y Gestión Escolar, no con la actividad de aula y las necesidades de allí derivadas.

\subsection{SOBRE EL PUESTO DE TRABAJO}

Los docentes más jóvenes acusan la presencia de una clara Inestabilidad Laboral, fundamentalmente aquellos que trabajan en Establecimientos Educacionales de Dependencia Particular Subvencionada. Esto lo relacionan directamente con el contexto de alta competencia en el que deben desenvolverse debido a la existencia de muchos Profesores/as de Historia y Ciencias Sociales. La Inestabilidad Laboral y el contexto de alta competencia los empuja a aferrarse a su Trabajo a pesar de que no se encuentran a gusto en el mismo muchas veces. Esta opción la explican por la búsqueda de seguridad laboral y la obligación asociada de aguantar.

Los docentes señalan que los sueldos percibidos son muy bajos y que sólo mejoran en la parte final de la Trayectoria Profesional, sin embargo, no todos tienen una visión negativa sobre sus remuneraciones, aunque estas sean muy bajas. Esta perspectiva la fundamentan en el argumento de que lo percibido alcanza para llevar a cabo el estilo de vida elegido. 
Sobre el Tiempo de su Jornada Laboral es posible señalar que la misma se organiza mayoritariamente en el desarrollo de Horas Lectivas. Esta situación es percibida por los docentes con malestar e impotencia, debido a que reconocen que afecta al desarrollo adecuado del Trabajo Pedagógico. Para poder realizar las actividades cotidianas en este marco los docentes señalan que han desarrollado estrategias de sobrevivencia que les permiten sortear la falta de Tiempo para preparar las múltiples actividades que tienen que realizar. Las extensas jornadas laborales también son sentidas como un abuso a los docentes y también a los estudiantes. Desde esta perspectiva, la forma en que los docentes se relacionan con el Tiempo se enmarca en la línea de una vinculación sociopolítica, en tanto la entienden como la expresión de su falta de poder para definir las condiciones en que se desarrolla su Trabajo, sienten así una falta de propiedad y control sobre su Tiempo y sobre sí mismo, lo que se manifiesta claramente en la irrupción constante de sus obligaciones laborales en su Tiempo Personal y Familiar.

\subsection{SOBRE EL TRABAJO DOCENTE EN CONTEXTO DE POBREZA}

Las motivaciones para desempeñarse en Contextos de Pobreza no son homogéneas; para algunos docentes responde a opciones políticas, para otros existiría una empatía de clase social y para otros este es un lugar en el que preferirían no desempeñarse.

Para la mayor parte de los docentes entrevistados, el fenómeno de la Pobreza es comprendido naturalmente, no ligado a las dinámicas sociales y económicas nacionales y globales, por lo mismo, la Pobreza se explicaría fundamentalmente como el resultado de malas opciones personales de cada individuo. Muy en esta línea, la imagen de los niños y jóvenes pobres con que se trabaja es muy negativa, siendo retratados discursivamente como malos pobres, incompletos, con bajas expectativas sobre sí mismo, "ubicados en el último peldaño", caracterizados por tener problemas de tipo cognitivo, sin saberes previos, en fin, impactados por las marcas materiales y simbólicas de la Pobreza. De esta manera, el desempeñarse en estos espacios implica a los docentes el desafío de enfrentarse cotidianamente a límites materiales y culturales, puesto que existiría una clara ausencia de recursos y la obligación de "partir de cero" constantemente en el desarrollo de aprendizajes de sus estudiantes.

\subsection{SOBRE EL TRABAJO DOCENTE}

Para las profesoras y profesores incluidos en esta investigación, el Trabajo Docente es posible de ser definido de muchas maneras, las que en algunos casos son contradictorias y articulan una red multidimensional de imágenes sobre el propio Trabajo:

El Trabajo Docente se define como una actividad marcada por la Inseguridad y la Flexibilización. El docentes es visto como un "ser desechable", que vive una relación muy asimétrica con sus empleadores, que se desempeña de forma muy desigual según sea la dependencia del Establecimiento Educacional en que Trabaja, debido a que identifican en la Dependencia Municipal niveles básicos de protección a su labor que son inexistentes en la dependencia Particular Subvencionada. De esta manera, estas condiciones del Trabajo Docente empujan al profesorado a buscar de manera constante niveles mayores de seguridad material y simbólica con miras a sentirse coherentes y respetados para poder seguir dedicándose a la docencia. 
El Trabajo Docente también es definido como una Actividad Administrativa. Esta imagen se construye a través de las presiones que vivencian los docentes por cumplir con las necesidades múltiples que les plantean la Escuela y los Padres y Apoderados con que se relacionan. De esta forma, más allá de tener que preocuparse de forma central en las tareas pedagógicas, muchas veces están obligados a cumplir con las demandas de corte administrativo que le imponen los empleadores y que les exigen los Padres y Apoderados.

También es posible recoger una mirada del Trabajo Docente como Profesión en el discurso de los profesores. El profesionalismo es vinculado a la imagen de la responsabilidad, al resguardar una ética en el ejercicio de las diferentes funciones, al actuar en base a opciones racionales, al ser cumplidor, soportar las múltiples exigencias que les son planteadas como expresión del "querer" a los estudiantes. Desde esta perspectiva, la mirada construida sobre el profesionalismo aquí expuesta involucra elementos relacionados con la vocación y la intensificación laboral, mezclados de tal manera que se constituye en un referente a seguir por parte de los docentes.

El Trabajo Docente también es definido por los docentes como una actividad "Denigrada", debido a que estos se ven obligados a participar en situaciones reñidas con la ética tales como falsear la asistencia diaria, la obligación de tener que cumplir con actividades diferentes a las pedagógicas y hacer mal el Trabajo debido a la falta de condiciones adecuadas. De esta manera, el Trabajo Docente se muestra aquí como una actividad que produce culpabilidad a los docentes.

El Trabajo Docente también es definidos por estos profesores como un Trabajo Asalariado, puesto que comprenden la necesidad de desplegar intentos serios y organizados por incidir en la configuración de su propio Trabajo.

Otra definición sobre el Trabajo Docente entregada por las y los profesores entrevistados es la de una Actividad Solitaria, puesto que estos asumirían que la responsabilidad de los resultados de su accionar es finalmente individual; además, por ausencia de tiempo, reconocen trabajar de manera independiente y desvinculada con respecto a los colegas, aún más, señalan que no existe diálogo pedagógico, didáctico o disciplinar con los colegas. Esta situación da cuenta de un estado de fragmentación profunda entre los docentes y consigo mismo en el Trabajo Docente, puesto que no estarían aseguradas las condiciones para abordar dialógica y reflexivamente lo que se hace de forma cotidiana.

La Rutinización y Mecanización es otra imagen del Trabajo Docente planteada por estos docentes, puesto que definen a su actividad en base a la idea de rutina constante articulada en base a actividades irreflexivas. El agobio y el aburrimiento también se constituirían en elementos que componen esta imagen del Trabajo Docente debido a que primaría la falta de novedad y las ansias de "estar en otro "lugar".

Finalmente, el Trabajo Docente es definido como una Actividad en Disputa, debido a que se desarrollaría en un contextos donde existirían amenazas externas constantes y además donde se desarrolla la fricción irresoluta entre la valorización del Tiempo Laboral en oposición al Tiempo Personal.

\subsection{SOBRE SER PROFESOR/A DE HISTORIA Y CIENCIAS SOCIALES}

Las y los Profesores de Historia y Ciencias Sociales tienen una comprensión de la Historia Escolar como una "Disciplina Ventajosa", debido a que se trata de un ámbito del conocimiento caracterizada por su actualidad y flexibilidad, lo que la hace propicia para 
vincularla con el presente. Para que esta propiedad pueda desarrollarse, estos docentes reconocen la necesidad de romper con las "formas añejas" de la enseñanza de la Historia.

Estos docentes también comparten una mirada de la asignatura de Historia y el Trabajo de Profesora o Profesor de Historia como una instancia y actor de carácter formativos potentísimos orientados al fortalecimiento de conocimientos y habilidades de base de los estudiantes, que incluso les servirá en su futuro laboral. Aún más, reconocen a esta instancia, la clase de Historia, como la única instancia formativa de la Cultura a la que pueden acceder los estudiantes.

También definen al Profesor/a de Historia como un Actor Político instalado en la Escuela, que como tal le correspondería jugar un rol fundamental en la formación de las nuevas generaciones. No obstante lo anterior, se reconoce también la necesidad de "omitir" en algunos contextos las posturas personales para sobrevivir en la Escuela.

\subsection{SOBRE EL TRABAJO DOCENTE Y LAS DIMENSIONES DEL TRABAJO DE MARX:}

Con respecto a la dimensión de Instrumentalidad, el Trabajo Docente es visualizado como una actividad que tiene un piso material amenazante. También es visualizado como una actividad "frontal" que permite vivenciar un proceso de rejuvenecimiento y enriquecimiento personal al conocer otras realidades distintas a las propias.

En el caso de la dimensión de Socialidad el Trabajo Docente fomentaría por una parte la "Soledad" y la "Fragmentación" con los pares, mientras que por otra parte, permitiría el desarrollo de relaciones abiertas y estimulantes con los estudiantes: lo que finalmente los "sostendría" humanamente en el desarrollo de su Trabajo.

En el caso de la dimensión de Expresividad, el Trabajo Docente le produce felicidad a los docentes al presenciar y ayudar en el crecimiento de los estudiantes, al ser reconocidos por los estudiantes, al poder desplegar los gustos personales en su actividad. Estos docentes también señalan sentirse realizados al poder llevar a cabo su labor con autonomía. Finalmente, algunos reconocen con respecto a esta dimensión, la necesidad de construir un oficio práctico que les permita ser mesurados con sus expectativas y valorar lo que se tiene.

8.7. SOBRE EL HABITUS CONSTRUIDO POR LOS PROFESORES DE HISTORIA Y CIENCIAS SOCIALES SOBRE EL TRABAJO DOCENTE EN CONTEXTOS DE POBREZA:

Es posible señalar que las y los Profesores de Historia y Ciencias Sociales incluidos en la presente investigación han construido un Habitus caracterizado por los siguientes elementos:

a) El Desacople radical entre el saber Histórico y Social como herramientas de comprensión del Contexto de Pobreza en el cual se trabaja.

b) Una forma de comprender la enseñanza de la Historia y las Ciencias Sociales en la instancia educativa de una forma única, homogénea e independiente del contexto.

c) Una forma de comprender al Sujeto Docente en un contexto de desagregación de sí mismo.

d) Una comprensión de la realidad del Trabajo Docente en un contexto de competencia desatada.

e) La idea de la dificultad e incapacidad de realizar acciones de carácter transformador. 


\section{CONCLUSIONES}

El Habitus construido por las y los Profesores de Historia y Ciencias Sociales que se desempeñan en Contextos de Pobreza muestra a un sujeto que vive su experiencia laboral de una forma radicalmente tensionante, que lo lleva a desatender y no relacionar sus propias necesidades y conocimientos construidos con miras a sentirse valorado y aceptado por un Sistema Educacional y Social que demanda mucho de él. De esta forma, este sujeto debe avanzar en un contexto material y simbólico adverso donde se debate entre la necesidad de realizar cotidianamente una performance de tipo auto-disciplinante y la necesidad de llevar a cabo acciones de auto-liberación y empoderamiento. Es en esta actuación dicotómica en que los Profesores de Historia y Ciencias Sociales avanzan en su Trayectoria Profesional, cargando las causas de sus decisiones y las insignias que la Sociedad cuelga de su estampa.

\section{PROYECCIONES}

Los resultados y conclusiones de esta investigación presentadas hasta aquí exhiben las siguientes preocupaciones y posibilidades que se proyectan más allá de los alcances de la misma:

a) Incorporar en la discusión de la Formación Inicial Docente de una forma más directa el debate sobre el Trabajo Docente y el Puesto de Trabajo.

b) Incorporar la perspectiva vital de los docentes en las políticas de configuración del Trabajo Docente.

c) Indagar más a fondo en la relación entre Trabajo y Placer/Realización en el ejercicio del Trabajo Docente.

d) Realizar estudios comparados más amplios sobre el Trabajo Docente a nivel latinoamericano con miras a una comprensión más acabada de la realidad de este en Chile y países afines o similares.

e) Incorporar la noción de Trabajo Docente y Puesto de Trabajo en cualquier discusión que intente abordar la "Calidad de la Educación".

f) Analizar las asimetrías existentes en el Trabajo Docente existente en el Sistema Municipal y el Particular Subvencionado y sus efectos en el éxito educativo.

\section{REFERENCIAS BIBLIOGRÁFICAS}

Bourdieu, P. (1997). Razones prácticas. Sobre la teoría de la acción. Barcelona: Anagrama.

CEPAL-UNESCO (1992). Educación y Conocimiento: eje de la transformación productiva con equidad. Santiago: Autor.

COLEGIO DE PROFESORES DE CHILE A. G. (2005). Estatuto Docente. Santiago: Autor.

Flick, U. (2004). Introducción a la investigación cualitativa. Madrid: Ediciones Morata-Fundación Paideia Galiza.

Goodson, I. (2004). Historias de vida del profesorado. Barcelona: Octaedro.

MINEDUC. (2009). Ley General de Educación. Santiago: Autor.

Noguera, J. (1998). La transformación del concepto de trabajo en la teoría social. Las aportaciones de las tradiciones marxistas. Barcelona: Universidad Autónoma de Barcelona. 
OCDE. (2009). Panorama de la Educación 2009. Indicadores de la OCDE. Madrid: Santillana Educación S. L.

Redondo, P. (2004). Escuelas y pobreza. Entre el desasosiego y la obstinación. Buenos Aires: Paidós. Santos, M. (2004). Por otra globalización. Del pensamiento único a la conciencia universal. Bogotá: Convenio Andrés Bello.

Tenti Fanfani, E. (2007). Profesionalización docente: consideraciones sociológicas. En Tenti Fanfani, E. (Comp.), El oficio de docente: vocación, trabajo y profesión en el siglo XXI (pp. 119-142). Buenos Aires: Siglo XXI Editores. 